\title{
Ideación suicida y su relación con la inteligencia emocional en universitarios colombianos
}

\author{
Suicidal ideation and its relationship to emotional \\ intelligence in Colombian college students
}

\author{
Yuly P Suárez $C^{1}$, Dayana E Restrepo $C^{2}$, Carmen C Caballero D ${ }^{1}$
}

Forma de citar: Suárez YP, Restrepo DE, Caballero CC. Ideación suicida y su relación con la inteligencia emocional en universitarios colombianos. Rev Univ Ind Santander Salud. 2016; 48(4): 470-478. DOI: http://dx.doi.org/10.18273/revsal. v48n4-2016005 (c) (i)

\section{RESUMEN}

Introducción: El suicidio es un problema de salud mundial que se encuentra en aumento, especialmente entre la población joven. Pensamientos sobre la muerte e ideas de quitarse la vida pueden presentarse de manera frecuente en estudiantes universitarios a lo largo del semestre académico por las nuevas experiencias de vida a los jóvenes, quienes se ven confrontados con importantes presiones sociales, personales, económicas y académicas. Algunos universitarios logran hacer frente a éstas demandas de manera efectiva mientras otros no. La habilidad emocional que permite responder con éxito ante experiencias adversas se le conoce como "inteligencia emocional". El presente estudio busca comprender de qué manera la aparición de ideas suicidas se encuentra relacionada con limitaciones en las habilidades emocionales de los universitarios colombianos para tolerar episodios de estrés. Objetivos: Identificar la relación entre ideación suicida e inteligencia emocional en jóvenes universitarios colombianos. Metodología: Estudio descriptivo correlacional, de diseño transversal. Se analizó la presencia de ideas suicidas en una muestra de 186 estudiantes universitarios, entre los 18 y 37 años de edad. Como instrumentos de medición se aplicaron los inventarios de ideación suicida de Beck y el Trait Meta Mood Scale-24. Se analizaron los datos en comparación a hallazgos previos en el tema del suicidio en en país. Resultados: El mantenimiento de ideas suicidas entre universitarios colombianos responde a dificultades de éstos jóvenes para comprender sus estados emocionales, reparar emociones negativas y afrontar problemas. Conclusión: las habilidades de inteligencia emocional son factores psicológicos que juegan un rol fundamental en la aparición y mantenimiento de ideas suicidas en los jóvenes universitarios colombianos.

Palabras clave: Suicidio, ideación suicida, emociones, inteligencia emocional, estudiantes.

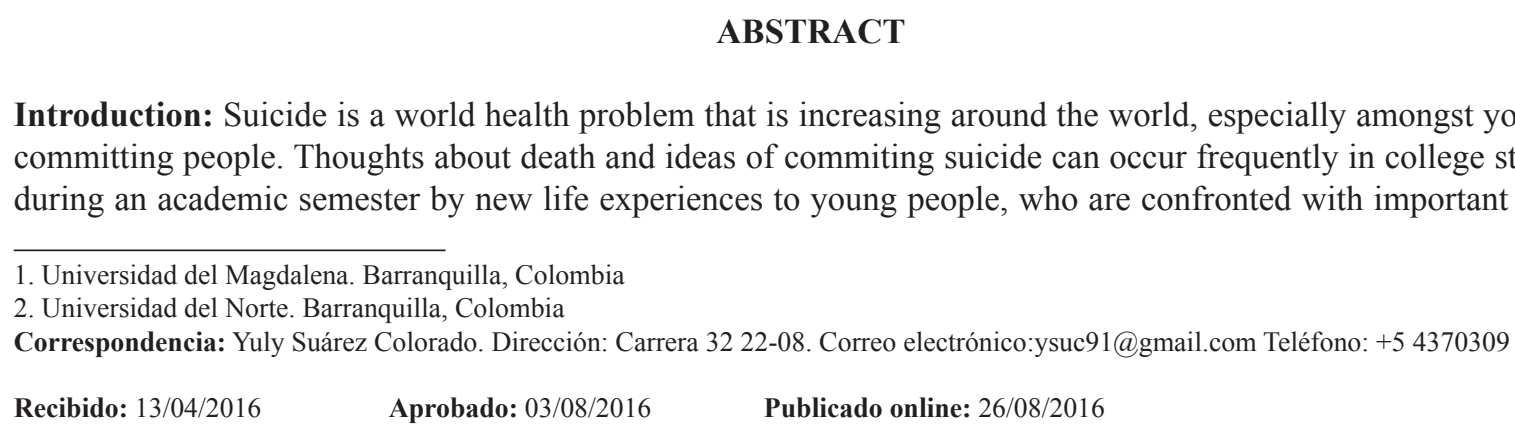


personal, economic and academic pressures. Some college students succeed by managing these demands effectively while others do not. The emotional competences that allow an individual to successfully cope with adverse situations are known as "emotional intelligence". The present research work seeks to gain some understanding about the way in which suicidal ideation is related to flaws in the emotional intelligence abilities for coping with adversity. Objectives: To identify the relationship between suicide ideation and emotional intelligence in Colombian college students Methodology: Correlational descriptive study, cross-sectional design. The presence of suicidal ideation was assessed for a sample of 186 college students between 18 and 37 years of age. The Beck Suicidal Ideation Inventory and the Trait Meta Mood Scale -24 were used as measurement instruments. Data analysis is presented in relation to previous findings of suicide studies in Colombia. Results: College students with difficulties for understanding their emotional states, for repairing their negative emotions and for coping with stressors are more likely to develop suicidal thoughts during the academic semester. Conclusion: Emotional intelligence skills are psychological factors that play a fundamental role in the development and maintenance of suicidal thoughts in young Colombian college students.

Keywords: Suicide, suicidal ideation, emotions, emotional intelligence, students.

\section{INTRODUCCIÓN}

Las organizaciones de salud alrededor del mundo durante los últimos 45 años han registrado incremento en las tasas de suicidio en todos los países, con especial vulnerabilidad población joven entre 10 y 24 años como el grupo etareo en mayor riesgo de incurrir en actos suicidas ${ }^{1-3}$. Aunque el comportamiento suicida representa un problema grave de salud pública mundial es un tema que no está recibiendo atención científica necesaria al ser limitada una estructura teórica que explique el desarrollo del comportamiento suicida, e insuficientes los programas validados para la prevención de éste fenómeno ${ }^{4}$.

Las ideas suicidas se definen como la predisposición cognitiva-afectiva de contemplar la idea de morirse y elaborar planes con el propósito de quitarse la vida ${ }^{5}$. La evaluación de la mayor o menor pretensión a llevar a cabo acciones suicidas se origina desde la ideación suicida pasiva, contemplación activa del propio suicidio, planeación y preparación, ejecución del intento suicida, y el suicidio consumado. En este conjunto de acciones, la relevancia de la ideación suicida radica en que representa un índice de riesgo para la muerte por suicidio. Un $20 \%$ de los sujetos que mantienen pensamientos suicidas más adelante reportarán intentos de suicidio 6 .

Al analizar la prevalencia de ideación suicida entre estudiantes universitarios, diversas investigaciones han identificado que los jóvenes presentan pensamientos suicidas frecuentes a lo largo del período académico ${ }^{7-9}$. Algunas explicaciones para este fenómeno señalan que el período universitario presenta importantes y nuevas experiencias de vida a los jóvenes, quienes se ven confrontados con nuevas presiones sociales y demandas personales como éxitos y fracasos académicos, mayor independencia de la familia, inicio y crisis de relaciones románticas, cambios en las metas de vida, nuevas amistades, entre otras. Estas experiencias resultan en algunas ocasiones abrumadoras y se acompañan de una percepción de escasos recursos para responder ${ }^{10,11}$.

Debido a la urgencia de intervenir el fenómeno del suicidio existe la necesidad de continuar investigaciones en población joven con el objetivo de identificar los factores psicológicos que protegen o representan riesgo de suicidio para los individuos. En este sentido, se ha afirmado que en las diferencias de los estilos de respuesta ante experiencias adversas subyace algún tipo de "habilidad y/o competencia psicológica" que permite a unos individuos adaptarse y afrontar las demandas del ambiente de manera efectiva mientras a otros no, a esta habilidad/competencia se denominaría inteligencia emocional ${ }^{12}$.

El rol de la inteligencia emocional en el funcionamiento psicológico de los individuos ha sido extensamente documentado. Es un hecho comprobado cómo el uso inteligente de las emociones, favorece la resolución de problemas, la toma de decisiones, la regulación del comportamiento propio ${ }^{13-15}$, el alcance de logros personales y profesionales ${ }^{16,17}$, el desempeño social exitoso ${ }^{13}$ y sentimientos de satisfacción ante la vida ${ }^{18,19}$. En especial se ha logrado establecer asociaciones entre las habilidades de inteligencia emocional con la salud mental y física-somática ${ }^{12,13,20-23}$.

Desde el modelo escalar o jerárquico de Mayer, et al. y Salovey, et al. las habilidades de inteligencia emocional son reconocidas como: percepción de emociones, 
Ideación suicida y su relación con la inteligencia emocional en universitarios colombianos

facilitación o asimilación emocional, comprensión emocional y regulación emocional ${ }^{24,25}$. De acuerdo a Rahgozar, et al., para llegar a la regulación emocional debe transitarse en un proceso lineal-consecutivo en donde se requiere de un determinado nivel de atención a los sentimientos para llegar a comprender los estados emocionales y por consiguiente regular las emociones; aunque existe la posibilidad que individuos con estilos rumiativos se resistan a la comprensión, la gestión y la expresión de sus emociones haciéndose más susceptibles a la ideación suicida ${ }^{26}$.

Un creciente número de trabajos investigativos señalan el rol de la inteligencia emocional como el principal factor protector contra la ideación suicida e intentos de suicidio en la adolescencia y adultez temprana. Éstos estudios han logrado demostrar cómo la aparición de ideas y comportamientos suicidas se encuentran relacionadas con limitaciones en las habilidades emocionales de los jóvenes para tolerar la experiencia de emociones negativas, dificultades para recuperarse de episodios de estrés emocional y escasas estrategias para afrontar las adversidades s,9,12,27-32 $^{\text {. }}$

Principalmente la dificultad en la atención emocional es predictiva de la ideación suicida. Elevados puntajes en atención emocional se han relacionado con susceptibilidad emocional y riesgo de suicidio entre estudiantes universitarios ${ }^{7,29-31}$. Igualmente, la dificultad para expresar emociones ha sido asociada con elevados síntomas depresivos e ideación suicida en los jóvenes ${ }^{10,29,32}$. En contraste, niveles elevados de claridad emocional y reparación emocional se relacionan con un buen ajuste emocional y mayor satisfacción ante la vida ${ }^{29-31,33,34}$. Específicamente, la claridad emocional garantiza un buen desempeño académico a los jóvenes a los jóvenes en su transición durante la vida universitaria ${ }^{13,15,35-37}$.

Teniendo en cuenta el riesgo que la ideación suicida representa para la consumación del acto suicida y el impacto que la inteligencia emocional tiene en la salud mental surge la pregunta ¿cuál es la relación existente entre las habilidades de inteligencia emocional como atención, claridad, reparación emocional y la ideación suicida entre en jóvenes universitarios colombianos?.

Se espera que la ideación suicida se encuentre relacionada con las dificultades para atender, comprender y reparar las emociones, así como las características inmersas en el pensamiento suicida, tales como la actitud hacia la muerte, deseo suicida, proyecto del intento, preparación del intento proyectado, entre otros factores de fondo, se relacionan con estas habilidades de inteligencia emocional.

\section{METODOLOGÍA}

La presente es una investigación de tipo descriptivacorrelacional de corte transversal ${ }^{27,38}$.

La elección de la muestra se realizó bajo el tipo no probabilístico intencional o por conveniencia, en donde el investigador determina la representatividad subjetivamente $^{38,39}$. La muestra estuvo formada por 186 estudiantes de administración de empresas en una Universidad Pública de Santa Marta (Colombia) quienes se encontraban cursando entre 3er y10mo semestre ( $M=5.2 \mathrm{DT}=2.2)$. El 42.5\% (45) de los sujetos eran hombres y el $57.5 \%$ (49) eran mujeres. Sus edades estuvieron comprendidas entre 18 y 37 años $(M=21.4$ $\mathrm{DT}=2.8$ ).

Para la medición de las variables de estudio se utilizaron los siguientes instrumentos:

Escala Rasgo de Meta-Conocimiento Emocional o Traid Meta Mood Scale 24-TMMS40. Evalúa el metaconocimiento de los estados emocionales a través de los componentes: atención emocional, claridad emocional y reparación emocional. El TMMS está conformado por 24 ítems con opción de respuesta tipo likert desde (1) nada de acuerdo a (5) totalmente de acuerdo. Sumados los ítems de cada componente se genera una puntuación que indicará si el sujeto debe mejorar, es adecuada o conserva excelente habilidades emocionales, a su vez esta clasificación se encuentra condicionada por el género.

La escala original validada y adaptada al español demostró alfa de Cronbach por encima de 0.85 en todos los componentes ${ }^{40}$.

Inventario de ideación suicida de Beck-ISB o Scale for Suicide Ideation $^{41}$ : Evalúa la recurrencia de pensamientos suicidas. El ISB consta de 19 reactivos y dos ítems adicionales de tipo descriptivo, en donde se informa del nivel de intensidad/seriedad que mejor reflejen las características de la ideación suicida. Si la puntuación es 0 en los ítems (4) Deseo de intentar suicidarse y (5) Intento pasivo de suicidarse, se omiten las secciones siguientes y se suspende el cuestionario, codificando como: "No aplicable", en caso contrario, se sigue aplicando. Sumados los ítems se considera que una puntuación igual o mayor que 1 es indicativa de riesgo de suicidio, a valores más altos se incrementa el riesgo. 
Salud Vol.48 No.4 Octubre - Diciembre de 2016

La escala demostró de alfa de cronbach en un 0.84 en estudiantes universitarios mexicanos ${ }^{42}$ para la escala total y en las subescalas I. Características de sus actitudes hacia la vida y la muerte: 0.90 , subescala II. Características de los pensamientos y los deseos de suicidarse: 0.45-0.65, subescala III. Características del proyecto de intento suicida: 0.61 , subescala IV. Realización del intento proyectado: 0.45 .

En el presente estudio se encontró adecuada consistencia para la escala de inteligencia emocional e ideación suicida. Tabla 1.

TABLA 1. Fiabilidad de la Escala Rasgo-Metaconocimiento Emocional e Inventario de Ideación Suicida.

\begin{tabular}{lcc}
\hline Variable & Componente & $\begin{array}{c}\text { Alfa de } \\
\text { Cronbach }\end{array}$ \\
\hline \multirow{2}{*}{$\begin{array}{l}\text { Inteligencia } \\
\text { Emocional }\end{array}$} & Atención emocional & 0.82 \\
& Claridad emocional & 0.81 \\
& Reparación emocional & 0.81 \\
& Total Ítems & 0.83 \\
Ideación & Deseos de suicidarse & 0.72 \\
Suicida & Proyecto de intento suicida & 0.71 \\
& Preparación del intento proyectado & 0.72 \\
& Total Ítems & 0.89 \\
\hline
\end{tabular}

La aplicación de los instrumentos se realizó en las aulas de clases bajo con el consentimiento informado de los estudiantes y previa autorización a los directivos del programa académico. Seguidamente se procedió a digitar la información en la base de datos y ejecutar el análisis a través del programa estadístico Statistical Package for the Social Sciences (SPSS 19.0).

En el procesamiento estadístico en el SPSS se utilizó la prueba paramétrica de Pearson para estimarlas correlaciones. Finalmente, el valor de $r$ positivo fue interpretado como relación lineal y el valor de R negativo como relación inversa. Los niveles de significancia menores a 0,01 y 0,05 demostraron aceptación de la hipótesis de investigación.

\section{RESULTADOS}

Se evidenció un perfil de características en habilidades emocionales de los universitarios: para el componente atención a las emociones la muestra manifiesta de manera relevante poca y demasiada atención sobre sus emociones, es decir, debe mejorar en la habilidad para atender, reconocer, identificar las emociones, aunque sobresalen universitarios con excelente habilidades de atención emocional $(\mathrm{M}=27.9$ y $\mathrm{DT}=5.2)$. Para el componente de claridad sobre las emociones deben mejorar una proporción menor de estudiantes, teniendo en cuenta que representativamente expresan adecuada y excelente habilidad de claridad emocional $(\mathrm{M}=30.0$ $\mathrm{DT}=5.0$ ). Finalmente, en reparación emocional se encuentra un estado adecuado y excelente, no obstante, una proporción debe mejorar su destreza para regular las emociones $(\mathrm{M}=32.7 \mathrm{DT}=4.8)$. Figura 1.
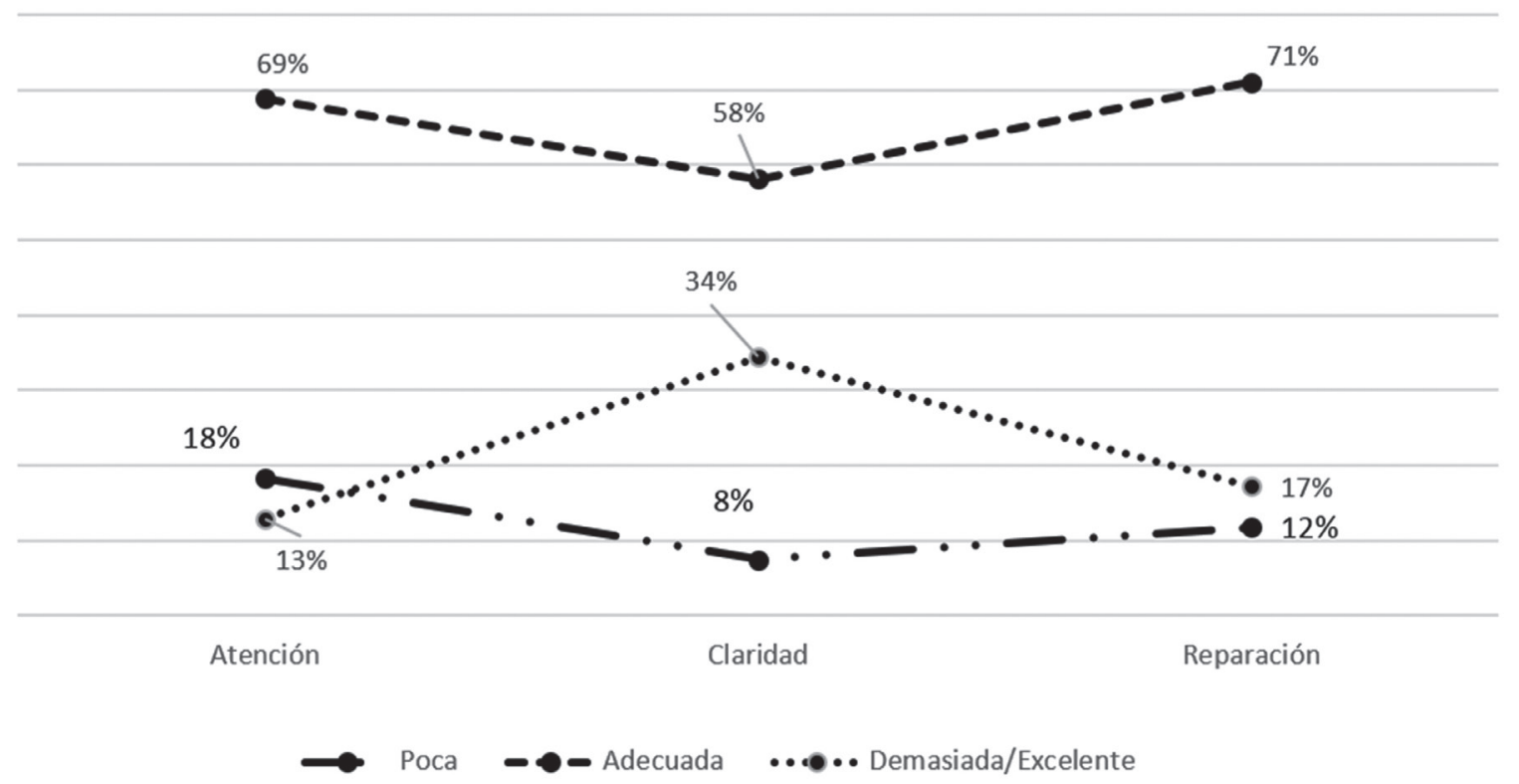

FIGURA 1. Habilidades de Inteligencia Emocional en Universitarios Colombianos. 
Ideación suicida y su relación con la inteligencia emocional en universitarios colombianos

Por otra parte, al identificar la ideación suicida se encontró en un 92,5\% (172) ausencia de ideación, este porcentaje obtuvo una puntuación de 0 en la escala total; no obstante, quienes puntúan de uno (1) en adelante presentan algún riesgo. Es decir, la presencia del riesgo desde los pensamientos suicidas se observa en el 7,5\% (14) de la muestra al momento de ser evaluados.

A través del estadístico Pearson, se encontró correlación negativa significativa entre la claridad emocional e ideación, a un nivel de 0,01 bilateral, indicando que entre mayor claridad emocional, menor es la ideación suicida. Así mismo, correlación negativa significativa entre reparación emocional e ideación suicida, a un nivel de 0,05 bilateral, señalando que entre mayor reparación emocional menor es la ideación suicida. Es decir individuos con dificultades en tener claridad y con dificultades para la reparación emocional, frecuentemente presentan ideas suicidas. No obstante, la habilidad para atender a las emociones no se encuentra relacionada con las ideas suicidas. Tabla 2.
TABLA 2. Ideación suicida y su relación con la inteligencia emocional.

\begin{tabular}{lcc}
\hline \multirow{2}{*}{ Inteligencia Emocional } & \multicolumn{2}{c}{ Ideación Suicida } \\
\cline { 2 - 3 } & $\mathbf{R}$ & Sig. \\
\hline Atención emocional & 0.108 & 0.143 \\
Claridad emocional & $-0.198^{* *}$ & 0.007 \\
Reparación emocional & $-0.170^{*}$ & 0.020 \\
\hline
\end{tabular}

Además, se identificaron correlaciones negativas significativas a un nivel de 0,05 bilateraln entre la actitud hacia la muerte, deseo suicida, proyecto del intento, preparación del intento proyectado, otros factores de fondo y la claridad y reparación. Esto indica que a menor claridad emocional, aumenta la probabilidad de que los individuos manifiesten mayor actitud hacia la muerte, mayor deseo suicida, incremento del proyecto de intento, y de la preparación cognitiva del intento proyectado; del mismo modo ocurre con la reparación emocional, exceptuando la actitud hacia la muerte, que no se relacionan con esta habilidad. Tabla 3.

TABLA 3. Relación entre las características de ideación suicida e inteligencia emocional.

\section{Ideación Suicida}

\begin{tabular}{lcccccc}
\multicolumn{1}{c}{$\begin{array}{c}\text { Inteligencia } \\
\text { Emocional }\end{array}$} & Actitud & $\begin{array}{c}\text { Hacia la } \\
\text { Muerte }\end{array}$ & $\begin{array}{c}\text { Deseo } \\
\text { Suicida }\end{array}$ & $\begin{array}{c}\text { Proyecto } \\
\text { del Intento }\end{array}$ & $\begin{array}{c}\text { Preparación } \\
\text { Intento Proyectado }\end{array}$ & $\begin{array}{c}\text { Factores } \\
\text { de Fondo }\end{array}$ \\
\hline Atención & $\mathrm{r}$ & 0.072 & 0.078 & 0.041 & 0.097 & 0.120 \\
Emocional & $\mathrm{Sig}$ & 0.330 & 0.290 & 0.579 & 0.187 & 0.104 \\
Claridad & $\mathrm{r}$ & $-0.158^{*}$ & $-0.202^{* *}$ & $-0.181^{*}$ & $-0.170^{*}$ & -0.121 \\
Emocional & $\mathrm{Sig}$ & 0.031 & 0.006 & 0.013 & 0.020 & 0.101 \\
Reparación & $\mathrm{r}$ & -0.096 & $-0.150^{*}$ & $-0.208^{* *}$ & $-0.162^{*}$ & -0.136 \\
Emocional & $\mathrm{Sig}$ & 0.195 & 0.041 & 0.004 & 0.027 & 0.064 \\
\hline
\end{tabular}

\section{DISCUSIÓN}

Esta investigación evidencia que la inteligencia emocional, específicamente la habilidad para comprender estados emocionales propios y reparar emociones negativas es un factor psicológico relacionado con la ideación suicida en universitarios de Colombia.

En general, los universitarios encuestados muestran adecuado estado en las habilidades emocionales como atención emocional, claridad emocional y reparación. Este hallazgo es congruente con los resultados de otros estudios realizados en Colombia con población universitaria ${ }^{43-45}$. Es decir, estos estudiantes tienen en cuenta sus estados de ánimo, pueden sentirse menos abrumados por sus emociones, tienen sensibilidad para identificarlas y monitorearlas, otorgan significado a las experiencias emocionales procesando el estado de ánimo adecuadamente, y por consiguiente recurren fácilmente a estrategias activas de afrontamiento ${ }^{46}$.

En cuanto a la presencia de ideación suicida, se registró un riesgo del $7.5 \%$, en comparación con otros estudios en universitarios colombianos: un $5.1 \%$ en Santa Marta, $41 \%$ en Caldas, $18 \%$ en estudiantes escolares y universitarios de Pasto, $31 \%$ en Tunja, $4.45 \%$ al $13 \%$ en Bogotá, y 16\% en Medellín ${ }^{47-52}$.

Por otra parte, las correlaciones encontradas señalan que la habilidad para tener claridad sobre las emociones y repararlas, anteponiendo a estados negativos emociones positivas están asociadas con la ideación suicida, indicando que a menor claridad emocional y 
menor reparación emocional, mayor es el riesgo desde la ideación suicida; particularmente, estas habilidades emocionales también trascienden relacionándose negativamente con actitud hacia la muerte, deseos suicidas, proyecto del intento y la realización del intento. Estos resultados sugieren la particularidad del procesamiento de la información emocional en sujetos con ideas de autolesión, coherente con diversos estudios que muestran un comportamiento diferencial de la inteligencia emocional en solución de problemas, felicidad, tolerancia al estrés y autorealización entre grupos con intento o ideación suicida y el grupo control ${ }^{53}$, un comportamiento diferencial en la atención, claridad y reparación entre grupos de universitarios con y sin ideación suicida ${ }^{54}$, un vínculo protector de la inteligencia emocional con la ideación e intentos suicidas en sujetos víctimas de abuso sexual ${ }^{12}$ y un vínculo predictor de las dificultades en emociones y habilidad para resolver problemas sobre los intentos suicidas en adolescentes y jóvenes ${ }^{55}$. Se resalta que se ha registrado antes la relación entre la habilidad para repararse o recuperarse emocionalmente y la ideación suicida ${ }^{48}$, esta misma habilidad ha estado correlacionada positivamente en varios estudios con la claridad emocional ${ }^{43,48,56-58}$, de manera que los hallazgos del presente estudio no son aislados al funcionamiento o despliegue de habilidades emocionales en los individuos.

La inteligencia emocional como capacidad para discriminar emociones y aprovecharlas para direccionar el comportamiento tiene un relevante funcionamiento en el bienestar psicológico sobre todo al momento de reparar heridas emocionales ${ }^{7}$, influye sobre el mantenimiento de los estados de ánimo positivos y reparando el afecto luego de experiencias negativas ${ }^{59}$. En contraste, una falta de conciencia de la emoción e inhabilidad para manejar las propias emociones son síntomas prevalentes en algunos desordenes de personalidad y del control de los impulsos ${ }^{60}$, que se conocen vinculados a los intentos de suicidio.

Desde otra perspectiva, las dificultades en inteligencia emocional tienen una importante influencia en los factores de riesgo ambientales, sociales, de personalidad y cognitivas que predisponen al suicidio, es decir, el inadecuado estado en habilidades emocionales haría vulnerable al sujeto a los factores de riesgo del suicidio y estos así mismos incrementan la aparición de pensamientos, intentos y eventos consumados ${ }^{61}$. Mientras, desde una vista sociológica, se resalta que el suicidio surge tras la desintegración y desregulación social, en este sentido la poca o demasiada integración social o regulación de las emociones por las normas culturales propician la consumación del suicidio ${ }^{62}$.
Finalmente, puede reconocerse a la inteligencia emocional como una habilidad con valor empírico para predecir la salud mental en los individuos ${ }^{63,64}$. Pero además, es real su vínculo con la salud psicosomática y la salud física ${ }^{65}$. De modo que en los procesos para el mantenimiento de la salud psicológica y física debe reconocerse las habilidades para la percepción de emociones, el uso de la información emocional para dirigir las cogniciones, la comprensión de la emoción y la regulación como contribuyentes ${ }^{59,65,66}$.

El estudio aunque presenta limitaciones para su generalización, como el tamaño de la muestra, participantes de una sola carrera universitaria y la evaluación de la inteligencia emocional a través de instrumentos de autoinforme, amplía el conocimiento existente acerca del problema social del suicidio. Igualmente aumenta la comprensión existente acerca de los procesos psicológicos que pueden reducir el riesgo de comportamientos suicidas.

Los resultados de esta investigación sugieren la necesidad de promover programas de entrenamiento en habilidades emocionales para los jóvenes con el objetivo de fortalecer sus habilidades para manejar situaciones de estrés académico, hacer frente a las dificultades de la vida, identificar, manejar y recuperarse de emociones dolorosas usando estrategias internas que interrumpan la trayectoria hacia el suicidio. Sin embargo, no se desconoce la función protectora de la religiosidad, resiliencia, autoestima, apoyo social, estilos de afrontamiento, estilo atribucional, esperanza y optimismo ${ }^{67,71}$.

Se recomienda direccionar las investigaciones hacia estudios comparativos con sujetos clínicos o con exposición previa a situaciones adversas y sujetos sin ninguna condición de riesgo a la enfermedad mental; en el caso de estudiantes universitarios sería importante conocer el estado de estas variables en época de exámenes y fuera de época de exámenes, reconociendo su curso longitudinal.

\section{CONSIDERACIONES ÉTICAS}

Este estudio adopta las consideraciones para la investigación en salud mental de la Resolución 8430 de 1993 del Ministerio de Salud, y los aspectos éticos en la investigación con humanos de la Ley 1090 de 2006 o Ley del Psicólogo en Colombia: secreto profesional, derecho a la no participación y retiro, consentimiento informado, devolución de resultados, anonimato. Este tipo de investigación no representa riesgo para la salud de los participantes. 


\section{CONFLICTO DE INTERESES}

Los autores manifiestan no tener ningún conflicto de intereses.

\section{REFERENCIAS}

1. Czyz EK, Horwitz A G, Eisenberg D, Kramer A, King C A. Self-reported barriers to professional help seeking among college students at elevated risk for suicide. J Am Coll Health. 2013; 61(7): 398-406. DOI: 10.1080/07448481.2013.820731.

2. Nock MK, Deming CA, Fullerton CS, Gilman SE, Goldenberg M, Kessler R. et al. Suicide among soldiers: a review of psychosocial risk and protective factors. Psychiatry. 2013; 76(2): 97-125. DOI: 10.1521 psyc.2013.76.2.97.

3. Vignolo J, Henderson E, Vacarezza M, Alvarez C, Alegretti M, Sosa A. Análisis de 123 años de muertes por suicidio en el Uruguay. 1887 2010. Rev Salud Pública. 2013; 17(1): 8-18.

4. Van Orden KA, Witte TK, Cukrowicz KC, Braithwaite SR, Selby EA, Joiner Jr TE. The interpersonal theory of suicide. Psychol Rev. 2010; 117(2): 575-600. DOI: 10.1037/a0018697.

5. Beck AT. Depression: causes and treatment. Philadelphia: University of Pennsylvania Press; 1972.

6. Borges G, Orozco R, Medina Mora ME. Índice de riesgo para el intento suicida en México. Sal Pública Méx. 2012; 54(6): 595-606. DOI: 10.1590/S003636342012000600008 .

7. Aradilla-Herrero A, Tomás-Sábado J. The role of emotional intelligence in nursing. (1a Ed). New York, NY: Nova Sciences Publishers; 2011.

8. Ceballos G. Suárez Y. El suicidio. (1a ed). Santa Marta: Editorial Unimagdalena; 2012.

9. Dasgupta S, Hazra S. Does student's emotional intelligence play role in their suicidal ideation. Indian J Community Psychol. 2011; 7(1): 190-197.

10. Johnson VK, Gans SE, Kerr S, LaValle W. Managing the transition to college: Family functioning, emotion coping, and adjustment in emerging adulthood. J Coll Stud Dev. 2010; 51(6): 607-621. DOI: $10.1353 /$ csd.2010.0022.

11. Robins RW, Fraley RC, Roberts BW, Trzesniewski KH. A longitudinal study of personality change in young adulthood. J Pers. 2001; 69(4): 617-640. DOI: 10.1111/1467-6494.694157.

12. Cha C, Nock M. Emotional intelligence is a protective factor for suicidal behavior. J Am Acad Child Adolesc Psychiatry. 2009; 48(4): 422-430. DOI: 10.1097/CHI.0b013e3181984f44.
13. Brackett MA, Rivers SE, Salovey P. Emotional intelligence: implications for personal, social, academic, and workplace success. Soc Personal Psychol Compass. 2011; 5(1): 88-103. DOI: 10.1111/j.1751-9004.2010.00334.x.

14. Mayer JD, Salovey P, Caruso DR. Emotional intelligence: theory, findings, and implications. Psychol Inq. 2004; 15(3): 197-215. DOI: 10.1111/j.1751-9004.2010.00334.x.

15. Nasir M, Masrur R. An exploration of emotional intelligence of the students of IIUI in relation to gender, age and academic achievement. Bull Ed Research. 2010; 32(1): 37-51.

16. Del Valle ID, Castillo MÁ. Inteligencia Emocional: una revisión del concepto y líneas de investigación. Cuad Estudios Empresarial. 2010; 20: 107-126.

17. O’Boyle Jr EH, Humphrey RH, Pollack JM, Hawver $\mathrm{TH}$, Story PA. The relation between emotional intelligence and job performance: a meta-analysis. J Organ Behav. 2010; 32(5): 788-818. DOI: 10.1002/ job.714.

18. Akerjordet K, Severinsson E. Emotional intelligence: a review of the literature with specific focus on empirical and epistemological perspectives. J Clin Nurs. 2007; 16(8): 1405-1416. DOI: 10.1111/j.13652702.2006.01749.x.

19. Gardner K, Qualter P. Emotional intelligence and borderline personality disorder. Pers. Individ Dif. 2009; 47(2): 94-98. DOI: 10.1016/j. paid.2009.02.004.

20. Aradilla-Herrero A, Tomás-Sábado J, GómezBenito J. Associations between emotional intelligence, depression and suicide risk in nursing students. Nurse Educ Today. 2014; 34(4): 520-525. DOI: 10.1016/j.nedt.2013.07.001.

21. Miranda R. Nolen-Hoeksema S. Brooding and reflection: rumination predicts suicidal ideation at 1-year follow-up in a community sample. Behav Res Ther. 2007; 45(12): 3088-3095. DOI: 10.1016/j. brat.2007.07.015.

22. O'Connor RC, Noyce R. Personality and cognitive processes: self-criticism and different types of rumination as predictors of suicidal ideation. Behav Res Ther. 2008; 46: 392-401. DOI: 10.1016/j. brat.2008.01.007.

23. Schutte NS, Malouff JM. Emotional intelligence mediates the relationship between mindfulness and subjective well-being. Pers Individ Dif. 2011; 50: 1116-1119. DOI: 10.1016/j.paid.2011.01.037.

24. Mayer JD, Salovey P. What is emotional intelligence? In P. Salovey D. J, Sluyter. (Eds.), Emotional development and emotional intelligence: Educational implications. (pp. 3-31). New York: 
Basic Books. 1997.

25. Salovey P, Mayer JD. Emotional intelligence. Imagin Cogn Pers. 1990; 9: 185-211.

26. Rahgozar S, Motahari AA. Inhibitory role of emotional intelligence in committing suicide. Ind $\mathrm{J}$ Sci Technol. 2011; 4(11): 1601-1606.

27. Abdollahi A, Talib MA. Emotional intelligence as a mediator between rumination and suicidal ideation among depressed inpatients: the moderating role of suicidal history. Psychiatry Res. 2015; 228(3): 591597. DOI: 10.1016/j.psychres.2015.05.046.

28. Pisani AR, Wyman PA, Petrova M, Schmeelk-Cone K, Goldston D B, Xia Y, et al. Emotion regulation difficulties, youth-adult relationships, and suicide attempts among high school students in underserved communities. J Youth Adolesc. 2013; 42(6): 807820. DOI: $10.1007 / \mathrm{s} 10964-012-9884-2$.

29. Weinberg A, Klonsky ED. Measurement of emotion dysregulation in adolescents. Psychol Assess. 2009; 21(4): 616-621. DOI: 10.1037/a0016669.

30. Gratz KL, Roemer L. Multidimensional assessment of emotionregulation and dysregulation: development, factor structure, and initialvalidation of thedifficultiesin emotionregulationscale. J Psychopathol Behav Assess. 2004; 26(1): 41-54. DOI: 10.1023/B:JOBA.0000007455.08539.94.

31. Neumann A, Van Lier PA, Gratz KL, Koot HM. Multidimensional assessment of emotion regulation difficulties in adolescents using the difficulties in emotion regulation scale. Assessment. 2010; 17: 138-149. DOI: 10.1177/1073191109349579.

32. Silk JS, Steinberg L, Morris AS. Adolescents' emotion regulation in daily life: Links to depressive symptoms and problem behavior. Child Dev. 2003;74(6): $1869-880$. DOI: $10.1046 /$ j.14678624.2003.00643.x.

33. Fernández-Berrocal P, Extremera N. La inteligencia emocional y el estudio de la felicidad. Rev Interuniv Forma Profes. 2009; 66 (23,3): 85-108.

34. Thayer J, Rossy LA, Ruiz-Padial E, Johnsen BH. Gender differences in the relations hip between emotional regulation and depressive symptoms. Cognit Ther Res. 2003; 27(3): 349-364. DOI: 10.1023/A:1023922618287.

35. Austin EJ, Saklofske DH, Mastoras SM. Emotional intelligence, coping and exam-related stress in Canadian undergraduate students. Aust J Psychol. 2010; 62(1): 42-50.

36. Parker JD, Hogan MJ, Eastabrook JM, Oke A, Wood LM. Emotional intelligence and student retention: Predicting the successful transition from high school to university. Pers. Individ Dif. 2006; 41(7): 13291336. DOI: 10.1016/j.paid.2006.04.022.
37. Hernández-Sampieri R, Fernández-Collado, Baptista L. Metodología de la investigación. (4a ed.).México D.F: Mc Graw Hill. 2006.

38. Mateu E. Casal J. Tamaño de la muestra. Revista de epidemiología y Medicina Preventiva. 2003; 1 : 8-14.

39. Fernández-Berrocal $\mathrm{P}$, Extremera $\mathrm{N}$, Ramos N. Validity and reliability of the Spanish modified version of the trait meta-mood scale. Psychol Rep. 2004; 94: 751-55. DOI: 10.2466/pr0.94.3.751-755.

40. Beck A, Kovacs M, Weissman A. Assement of suicidal intent: the scale for suicide ideation. J Consult Clin Psychol. 1979; 47: 343-352.

41. González S, Díaz A, Ortiz S, González C, González J. Características psicométricas de la Escala de Ideación Suicida de Beck (ISB) en estudiantes universitarios de la ciudad de México. Sal Mental. 2000; 23(2): 21-30.

42. Rodríguez U, Amaya A, Argota A. Inteligencia emocional percibida y ansiedad de rasgo-estado en estudiantes de psicología de una universidad pública del Magdalena. Psicogente. 2011; 14(26): 310-320.

43. Rodríguez U, Suárez Y. Relación entre inteligencia emocional, depresión y rendimiento académico en estudiantes de psicología. Psicogente. 2012; 15(28): 348-359.

44. Contreras F, Barbosa D, Espinosa J. Personalidad, inteligencia emocional, y afectividad en estudiantes universitarios de áreas empresariales implicaciones para la formación de líderes. Diversitas: perspectivas en psicología. 2010; 6(1): 65-79. DOI: 10.15332/ s1794-9998.2010.0001.05.

45. Salguero JM, Extremera N, Fernández berrocal P. A meta mood model of rumination and depression: Preliminary test in a non clinical population. Scand J Psychol. 2013;54(2):166-72. DOI: 10.1111/ sjop. 12026.

46. Amezquita M, Gonzales R, Zuluaga D. Prevalencia de depresión e ideación suicida en estudiantes de $8,9,10$ y 11 grado, en ocho colegios oficiales de Manizales. Hacia la promoción de la salud. 2008; 13: 143-153.

47. Ceballos G, Suárez Y. Características de inteligencia emocional y su relación con la ideación suicida en una muestra de estudiantes de Psicología. Rev CES Psicol. 2012; 5(2): 88-100.

48. Sánchez R, Cáceres H, Gómez D. Ideación suicida en adolescentes universitarios: prevalencia $\mathrm{y}$ factores asociados. Biomédica. 2002; 22: 407-416.

49. Siabato Macías EF, Salamanca Camargo Y. Factors Associated with Suicidal Ideation in College Students. Psychologia. Avances de la Disciplina. 2015; 9(1): 71-81. 
50. Villalobos-Galvis F. Situación de la conducta suicida en estudiantes de colegios y universidades de San Juan de Pasto, Colombia. Sal mental. 2009; 31: $165-171$.

51. Blandón Cuesta OM. Carmona Parra JA, Mendoza Orozco MZ, Medina Pérez ÓA. Ideación suicida y factores asociados en jóvenes universitarios de la ciudad de Medellín. Rev Arch Méd Camagüey. 2015; 19(5): 469-478.

52. Ahmadian M, Shamshiri Nezam T, Badvee I, Homayouni A. Comparison the emotional intelligence components of suicidal patients and non-clinical samples [Abstract]. Eur Psychiatry. 2009; 24 (1):143-53. DOI: 10.1016/S09249338(09)71301-X.

53. Caballero C, Suárez Y, Bruges H. Características de inteligencia emocional en un grupo de universitarios con y sin ideación suicida. Rev CES Psicol. 2015; 8(2): 138-155.

54. Dour H, Cha C. Nock M. Evidence for an emotioncognition interaction in the statistical prediction of suicide attempts.Behav Res Ther. 2011; 49 (4): 29498. http://dx.doi.org/10.1016/j.brat.2011.01.010.

55. Anadón O. Inteligencia emocional percibida y optimismo disposicional en estudiantes universitarios. REIFOP. 2006; 9(1): 1-13.

56. Gartzia L, Aritzeta A, Balluerka N, Heredia EB. Emotional intelligence and gender: beyond sex differences. Anal Psicol. 2012; 28(2): 567-575. DOI: 10.6018 /analesps.28.2.124111.

57. Peña M, Rey L, Extremera N. Life satisfaction and engagement in elementary and primary educators: differences in emotional intelligence and gender. Rev Psicodidáct. 2012; 17(2): 341-358. DOI: 10.1387/RevPsicodidact.1220.

58. Schutte NS, Malouff JM, Simunek M, McKenley J, Hollander S. Characteristic emotional intelligence and emotional well-being. Cogn Emot. 2002; 16(6): 769-785. DOI: $10.1080 / 02699930143000482$.

59. Zeidner M, Roberts R D, Matthews G. Can emotional intelligence be schooled? A critical review. Educ Psychol. 2002; 37(4): 215-231. DOI: 10.1207/S15326985EP3704 2.

60. Suárez Y. La inteligencia emocional como factor protector ante el suicidio. Rev Psicol Gepu. 2012; 3(1): 182-200.

61. Durkheim E. El suicidio.(múltiples ediciones, primera edición). 1987.

62. Lanciano T, Curci A, Zatton E. Why do some people ruminate more or less than others? The role of Emotional Intelligence ability. Eur. J. Psychol. 2010; 6(2): 65-84. DOI: 10.5964/ejop.v6i2.185.

63. Salguero JM, Palomera R, Fernández-Berrocal P.
Perceived emotional intelligence as predictor of psychological adjustment in adolescents: a 1-year prospective study. Eur J Psychol Educ. 2012; 27(1): 21-34. DOI: 10.1007/s10212-011-0063-8.

64. Schutte NS, Malouff JM, Thorsteinsson EB, Bhullar N,Rooke S E. A meta-analytic investigation of the relationship between emotional intelligence and health. Pers Individ. Dif. 2007; 42(6): 921-933. DOI: doi.org/10.1016/j.paid.2006.09.003.

65. Kleiman EM, Liu RT. Social support as a protective factor in suicide: Findings from two nationally representative samples. J Affect Disord. 2013; 150(2): 540-545. DOI: 10.1016/j.jad.2013.01.033.

66. Kleiman EM, Miller AB, Riskind JH. Enhancing attributional style as a protective factor in suicide. J Affect Disord. 2012; 143(1-3): 236-40. DOI: 10.1016/j.jad.2012.05.014.

67. Mosqueiro BP, Da Rocha NS, Fleck MP de A. Intrinsic religiosity, resilience, quality of life, and suicide risk in depressed inpatients. J Affect Disord. 2015; 179: 128-133. DOI: 10.1016/j. jad.2015.03.022.

68. Gooding P, Tarrier N, Dunn G, Shaw J, Awenat Y, Ulph F, et al. The moderating effects of coping and self-esteem on the relationship between defeat, entrapment and suicidality in a sample of prisoners at high risk of suicide. Eur Psychiatry. 2015; 30(8): 988-994. DOI: 10.1016/j.eurpsy.2015.09.002.

69. Raymond P, Tucker, Wingate LR, O'Keefe VM, Mills A C, Rasmussen K, et al. Rumination and suicidal ideation: The moderating roles of hope and optimism. Pers Individ Dif. 2013; 55(5): 606-611. DOI: $10.1016 /$ j.paid.2013.05.013. 